\title{
Los servicios de fisioterapia domiciliaria en el sistema sanitario público de la Península Ibérica
}

\author{
R. López-Liria ${ }^{a}$ * , D. Padilla Góngora ${ }^{b}$, D.J. Catalán Matamoros ${ }^{c}$, M.I.M.P. Sousa Coutinho ${ }^{d}$, \\ A.M. da Silva Miranda y A.C. Correia Brito ${ }^{f}$
}

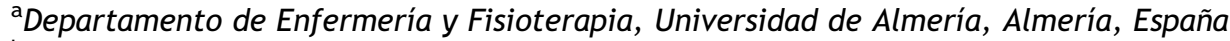

${ }^{\mathrm{b}}$ Facultad de Humanidades y Ciencias de la Educación, Universidad de Almería, Almería, España

${ }^{\mathrm{c}}$ Centro Europeo de Prevención y Control de Enfermedades, Suecia

${ }^{\mathrm{d}}$ Fisioterapia, Escola Superior de Tecnologia da Saúde Lisboa, Lisboa, Portugal

${ }^{\mathrm{e}}$ Fisioterapia, Escola Superior de Tecnologia da Saúde de Lisboa, Lisboa, Portugal

${ }^{\mathrm{f}}$ Fisioterapia, Escola Superior de Tecnologia da Saúde de Lisboa, Portugal

Recibido el 9 de enero de 2006; aceptado el 19 de enero de 2009

Disponible en Internet el 26 de junio de 2009

\section{PALABRAS CLAVE \\ Fisioterapia; Atención domiciliaria; Atención primaria}

\begin{abstract}
Resumen
Objetivos: Conocer los principales servicios de fisioterapia domiciliaria que oferta el Sistema Sanitario Público para las personas mayores dependientes de la Península Ibérica (España y Portugal).

Estrategia de búsqueda: Revisión bibliográfica actualizada y exhaustiva sobre los estudios previos; estancia de investigación en Hospitales, Centros de Salud y organismos financiados por la Administración Pública en ambos países; entrevistas en profundidad a informantes estratégicos.

Síntesis de los resultados: Se describe la situación de la fisioterapia domiciliaria en España, desde el año 1987 hasta agosto de 2008. Se detecta que en Portugal la fisioterapia domiciliaria está recogida en la cartera de servicios de atención primaria (Decreto-Ley $\mathrm{n}^{\circ}$ 28/2008, del 22 de febrero).

Conclusiones: Existen pocas investigaciones a nivel de la Península que ofrezcan datos objetivos basados en la evidencia científica sobre la efectividad de estos servicios de fisioterapia, la satisfacción del usuario y las repercusiones que producen con respecto a otros tratamientos; sin embargo, estos estudios son necesarios para la evaluación de las
\end{abstract}

\footnotetext{
*Autor para correspondencia.

Correo electrónico: rll040@ual.es (R. López-Liria).
} 


\section{KEYWORDS}

Physiotherapy;

Home care;

Primary care

políticas sanitarias, la distribución adecuada de recursos, la revisión de los programas, la metodología de actuación, etc.

(c) 2006 Asociación Española de Fisioterapeutas. Publicado por Elsevier España, S.L. Todos los derechos reservados.

\title{
Homecare physiotherapy in the iberian peninsula public health system
}

\begin{abstract}
Objectives: To become acquainted with the principal homecare physiotherapy services that are offered within the Public Health System for dependent elderly patients in the Iberian Peninsula (Spain and Portugal).

Methods: A thorough and up-dated review of the literature regarding previous studies, research stays in Hospitals, Primary Health Care Centers and Public Health institutions in both countries financed by the Public Health Administration; in-depth interviews with specific informants.

Results: The situation of homecare physiotherapy in Spain from the year 1987 to the August 2008 is described. In Portugal, these services are established within the services regulated by Primary Health Care (Decree-Law no. 28/2008, of February 22).

Conclusions: There are few studies in the Iberian Peninsula that offer scientific evidence based information on the effectiveness of these physiotherapy services, user satisfaction and the effect they have in relation to other treatments. However, these studies are needed in order to evaluate the current health legislations, appropriate distribution of resources, to review the clinical programs, clinical action methodologies, etc.

(c) 2006 Asociación Española de Fisioterapeutas. Published by Elsevier España, S.L. All rights reserved.
\end{abstract}

\section{Objetivos}

La fisioterapia domiciliaria es una práctica que se viene ejercitando desde hace muchos años, pero la integración de esta modalidad asistencial desde una perspectiva institucionalizada y estructurada, formando parte de los planes de intervención de los diferentes departamentos de sanidad en las administraciones públicas de la Península Ibérica, es relativamente reciente (en España se establece en el año 2000 el Concierto Marco para la Rehabilitación por parte del INSALUD, CMR Carrasco) ${ }^{1}$. El servicio surge ante la necesidad de aproximar y personalizar la asistencia a cada paciente e implicar a la familia en su proceso de recuperación en el hogar. Una mayor sensibilización de la comunidad hacia los beneficios de los cuidados en casa, de la sociedad científica y de los responsables políticos hace que esta prestación sanitaria tienda a desarrollarse de manera cada vez más uniforme en el mapa europeo de recursos sanitarios y sociosanitarios (Organización Mundial de la Salud, 2004) ${ }^{2}$.

La atención domiciliaria no tiene como objetivo sustituir una buena asistencia por otra menos buena pero más económica, sino que ésta surge en un momento determinado y con una función propia dentro del proceso de recuperación del paciente, demostrando que es una alternativa eficaz, eficiente $y$ de alta calidad frente a los tratamientos hospitalarios. No sólo mantiene la calidad del servicio, sino que se suma el beneficio de la tranquilidad del hogar, además del valor añadido que representa la intervención en un escenario real (Montagut et al, 2005) ${ }^{3}$.

En los casos en que existe una clara relación entre dependencia y déficit de movilidad es en los que se produce con mayor frecuencia la incapacidad para mantener al individuo en su hogar de un modo satisfactorio (IMSERSO, 2006) ${ }^{4}$. El mantenimiento de la funcionalidad en estos pacientes es fundamental para evitar hospitalizaciones e ingresos innecesarios en residencias. Cuando un anciano se encama como consecuencia de una enfermedad, es importante que el fisioterapeuta le ayude a recuperar rápidamente su movilidad, pues un tratamiento fisioterapéutico adecuado y adaptado a su estadio persigue alcanzar los niveles de función previos y evitar el deterioro de las funciones, potenciado las actividades de la vida diaria y buscando que su estado de salud sea el mejor posible. El tratamiento domiciliario maximiza su calidad de vida y la de sus allegados, favoreciendo de este modo el sistema informal de cuidado ${ }^{5,6}$.

Con la revisión que presentamos en este trabajo, deseamos conocer cuáles son los principales servicios de fisioterapia que son ofertados por el Sistema Sanitario Público para las personas mayores dependientes en la Península Ibérica, incidiendo específicamente en el campo de la fisioterapia domiciliaria a través de:

- Análisis exhaustivo sobre los estudios y la evidencia científica existente en relación con las intervenciones fisioterapéuticas realizadas en el domicilio dentro del Sistema Sanitario Público de la Península Ibérica.

- Historia de la fisioterapia domiciliaria dentro del Sistema Sanitario Público en España y Portugal.

- Realidad de la atención fisioterápica domiciliaria en la actualidad.

\section{Estrategia de búsqueda}

En primer lugar, se realizó una revisión bibliográfica actual y exhaustiva sobre los estudios previos en el ámbito de la 
Tabla 1 Descriptores, bases de datos y resultados de la investigación

\begin{tabular}{|c|c|c|}
\hline Español & Bases de datos & Resultados \\
\hline 1. «Fisioterapia» & CSIC IME & 452 \\
\hline 2. «Rehabilitación» & CSIC IME & 1.649 \\
\hline 3. «Atención domiciliaria» AND 1 & Dialnet & 2 \\
\hline \multirow[t]{4}{*}{ 4. «Atención domiciliaria» AND 2} & CSIC IME & 3 \\
\hline & CUIDEN Plus & 5 \\
\hline & Dialnet & 2 \\
\hline & CUIDEN Plus & 17 \\
\hline \multirow[t]{7}{*}{ 5. «Domicilio» AND 1} & CSIC IME & 5 \\
\hline & Dialnet & 6 \\
\hline & ENFISPO & 2 \\
\hline & Portal de & 2 \\
\hline & Mayores & \\
\hline & CUIDEN Plus & 6 \\
\hline & Cochrane Plus & 2 \\
\hline \multirow[t]{7}{*}{ 6. «Domicilio» AND 2} & CSIC IME & 11 \\
\hline & Dialnet & 9 \\
\hline & ENFISPO & 7 \\
\hline & Portal de & 17 \\
\hline & Mayores & \\
\hline & CUIDEN Plus & 19 \\
\hline & Cochrane Plus & 7 \\
\hline 7. 3 AND 4 AND 5 AND 6 & CSIC IME & 2 \\
\hline Portugués & Bases de datos & Resultados \\
\hline 1. «Fisioterapia» $A N D$ «Domicil*» $A N D$ «Portugal» & Scirus & 206 \\
\hline 2. «Reabilitacao» $A N D$ «fisioterapia» AND «Domicil*» AND «Portugal» & Scirus & 89 \\
\hline 3. «Fisioterapia» AND «Domicil*» AND «Portugal» ANDNOT (Bra?il OR universitat) & Scirus & 7 \\
\hline $\begin{array}{l}\text { 4. «Reabilitacao» AND «Fisioterapia» AND «Portugal» (Todos los campos y en } \\
\text { portugués) }\end{array}$ & Ovid & 0 \\
\hline Inglés & \# Bases de datos & Resultados \\
\hline 1. «Physical Therapy» $O R$ «Physiotherapy» & Ovid & 135.282 \\
\hline 2. «Rehabilitation» & Ovid & 262.142 \\
\hline 3. «Home care services» $O R$ «Home care» $O R$ «home-care-servided-hospital based» & & 57.978 \\
\hline 4. «Rehabilitation at home» & Ovid & 456 \\
\hline 5. «Portugal».af. & Ovid & 35.896 \\
\hline 6. «Portugal».cp. & Ovid & 9.380 \\
\hline 7. 1 OR 2 OR 4 & Ovid & 351.951 \\
\hline 8. 3 AND 5 AND 7 & Ovid & 16 \\
\hline 9. 6 AND 8 & Ovid & 0 \\
\hline Inglés & \# Bases de datos & Resultados \\
\hline 1. «Physical Therapy OR Physiotherapy» & Ovid & 135.282 \\
\hline 2. «Rehabilitation» & Ovid & 262.142 \\
\hline 3. «Home care services» $O R$ «Home care» $O R$ «home-care-servided-hospital based» & & 57.978 \\
\hline 4. «Rehabilitation at home» & Ovid & 456 \\
\hline 5. 1 OR 2 OR 4 & Ovid & 351.951 \\
\hline 6. (spain $O R$ spanish). af,cp. & Ovid & 504.441 \\
\hline 7. 3 AND 5 AND 6 & Ovid & 163 \\
\hline 8. (physical therapy $O R$ physiotherapy).ab,cp. & Ovid & 17.369 \\
\hline 9. (rehabilitation $O R$ «rehabilitation at home»).ab,cp. & Ovid & 73.512 \\
\hline 10. (spain $O R$ spanish).ab,cp. & Ovid & 178.120 \\
\hline $\begin{array}{l}\text { 11. («home care services» } O R \text { «home care» } O R \text { «home care serviced hospital } \\
\text { based»).ab,cp. }\end{array}$ & & 12.183 \\
\hline 12. 8 OR 9 & Ovid & 87.822 \\
\hline 13. 10 AND 11 AND 12 & Ovid & 1 \\
\hline 14. 11 AND 12 & Ovid & 521 \\
\hline 15. 6 AND 14 & Ovid & 2 \\
\hline
\end{tabular}

ab: abstract; af: all field; cp: country of publication 
fisioterapia y rehabilitación domiciliaria y el Sistema Sanitario Público, orientados a la población dependiente en España y Portugal, para sacar a la luz la importancia que tiene el abordaje y la investigación sobre los métodos y los procedimientos para ayudar a la población dependiente a alcanzar la máxima funcionalidad, así como los servicios que se le prestan a ésta y a sus familiares.

La elección de las palabras clave/descriptoras para realizar la búsqueda de información en inglés se realizó a través del MeSH Database de PubMed (o entre las opciones suggestions).

También se ha realizado una búsqueda con los descriptores en español, según las recomendaciones del Consejo Superior de Investigaciones Científicas de Biomedicina (CSIC IME) (búsqueda por índice) y en el idioma portugués.

En relación con los operadores voléanos, se seleccionó $A N D$ y $O R$. Las fechas de la búsqueda no se limitaron en antigüedad, abarcando hasta el 1 de agosto de 2008.

De los artículos resultantes de la búsqueda se revisaron los resúmenes para determinar si cumplían los criterios de inclusión para ser seleccionados (documentos obtenidos de los descriptores definidos y que respondiesen a los objetivos previos de este artículo).

Los descriptores utilizados para la búsqueda en las bases de datos se exponen en la tabla 1.

Se han consultado las siguientes bases de datos españolas: CUIDEN Plus, CSIC IME, ENFISPO, Dialnet y el Portal de Mayores (que contiene aproximadamente 16.500 referencias bibliográficas sobre Gerontología y Geriatría obtenidas de las bases de datos especializadas Cindoc, Clase, Lilacs, Periódica, TESEO, de los fondos del IMSERSO y de otras fuentes, y están organizadas siguiendo una clasificación propia. Recoge la producción española, latinoamericana y en otros idiomas sobre España desde 1975).

En Portugal, se utilizó el Centro de Documentación e información de la Escola Superior de Tecnología da Saude de Lisboa (Biblioteca Nacional Digital, EBSCO host, Academic Search Complete, American Chemical Society, base de dados de la plataforma Web of Knowledge, British Library, Directory of Open Access Journals, etc.), su archivo digital y sus publicaciones periódicas, y el Portal del Ministerio de Salud en Portugal, que contiene a su vez una biblioteca virtual de Salud.

Las bases de datos internacionales consultadas han sido Scirus (Open Access: NALTD, tesis, prepints, portales SD, Nature, Pubmed, BioMed Central, Wileylnterscience, etc.), Ovid (Medline, Cinahl, Journals@Ovid.com, CAB Health, Psycinfo, etc.), y Cocrhane Plus.

Además, se ha llevado a cabo un estudio descriptivo en una unidad móvil de rehabilitación y fisioterapia en la provincia de Almería, lo que nos ha ofrecido información in situ sobre los pacientes atendidos, procesos que demanda la fisioterapia, actuaciones llevadas a cabo y evolución del servicio en Andalucía ${ }^{7}$.

Se completó la información con una estancia de investigación en la ciudad de Lisboa durante dos meses, para conocer la realidad de este país, en cuanto a los recursos de fisioterapia existentes en los hospitales, los centros de salud y los organismos financiados públicamente por el Sistema de Salud Portugués, y con la colaboración de la Escola Superior de Tecnología da Saude de Lisboa, lo que nos ha dado la posibilidad de interactuar también con los fisioterapeutas portugueses en sus lugares de trabajo y conocer cuál es el estado real de la cuestión en el desarrollo de los servicios de fisioterapia.

Esto nos ha facilitado utilizar para este trabajo, como fuentes de información, la realización de entrevistas en profundidad a informantes estratégicos (fisioterapeutas y médicos rehabilitadores de atención primaria y especializada) para averiguar cuál es su conocimiento y visión sobre la fisioterapia domiciliaria presente y futura en ambos países.

\section{Selección de estudios y casos}

El análisis de los resultados encontrados, de la legislación y de todas las fuentes de información obtenidas a través de la metodología expuesta nos permite describir la situación de la fisioterapia y rehabilitación en el ámbito domiciliario en España, desde el año 1987 hasta la actualidad, como sigue:

El Departamento de Sanidad de la Generalitat de Cataluña en este año fue el pionero en poner en marcha un servicio de rehabilitación domiciliario financiado al $100 \%$ por la administración pública ${ }^{3}$. En el año 1999, el INSALUD llevó a cabo un proceso de reestructuración de los criterios que regulaban la rehabilitación en su área de influencia, incluyendo la rehabilitación domiciliaria, ambulatoria, respiratoria, cardiológica y logopédica. Toda la normativa se recogió en un documento llamado "Carrasco", concierto marco para procedimientos terapéuticos de rehabilitación: CMR-2000 ${ }^{1}$, que en la actualidad está siendo utilizado en las comunidades autónomas de Asturias, Castilla y León y Madrid, así como en la ciudad autónoma de Ceuta.

En el Plan de Rehabilitación de la Comunidad Valenciana (1988, 1991) de la Consellería de Sanitat ya se contempla, dentro de las líneas de actuación asistencial, la rehabilitación domiciliaria en sus páginas ${ }^{8,9}$. También en el ámbito público, el Plan de Rehabilitación de Castilla-La Mancha'04 elabora un documento con el apoyo de la Sociedad Española de Rehabilitación y Medicina Física que contempla la atención fisioterápica domiciliaria en uno de sus aparta$\operatorname{dos}^{10}$.

En el año 2002, la Junta de Andalucía establece el Servicio de Fisioterapia y Rehabilitación en Atención Primaria y los Equipos Móviles, integrándolos en el Plan de Apoyo a las Familias Andaluzas ${ }^{11}$, mejorando la accesibilidad para los pacientes y sus familias, ofertándolo en el domicilio, estableciendo un conjunto de medidas, servicios y ayudas que se reflejan en un apoyo a la institución familiar desde una perspectiva global ${ }^{12,13}$. La Consejería de Salud de la Junta de Andalucía cuenta actualmente con 186 salas de fisioterapia y rehabilitación en centros de salud y 46 equipos móviles, que se distribuyen por provincias de la siguiente manera: Almería (16 salas y 4 equipos móviles); Cádiz (22 salas y 10 equipos móviles); Córdoba ( 21 salas y 4 equipos móviles); Granada (24 salas y 4 equipos móviles); Huelva (15 salas y 4 equipos móviles); Jaén (24 salas y 4 equipos móviles); Málaga (29 salas y 6 equipos móviles), y Sevilla (35 salas y 10 equipos móviles).

En Portugal no hemos encontrado evidencias sobre servicios sanitarios públicos que ofrezcan específicamente la fisioterapia domiciliaria, excepto en la cartera de servicios de los fisioterapeutas de atención primaria, donde 
tienen contempladas diez horas semanales que pueden dedicar a esta atención si se precisa. Recientemente, se ha aprobado en este país el Decreto-Ley $n^{\circ} 28 / 2008$, del 22 de febrero $^{14}$, donde se reconoce a los cuidados de salud primarios como el pilar central del sistema de salud, constituyendo el primer acceso de los cuidados y prestaciones de salud, asumiendo importantes funciones de promoción de la salud y prevención de la enfermedad, prestando cuidados a la enfermedad y ligado a otros servicios para la continuidad de la atención ${ }^{15}$. Una de las principales novedades de la presente intervención legislativa consiste en la creación de los agrupamientos de centros de salud (ACES). Estas unidades funcionales constan $\mathrm{de}^{14}$ :

- Unidades de salud familiar (USF): donde no se contempla la figura del fisioterapeuta.

- Unidades de cuidados de salud personalizados (UCSP): médicos, enfermeros y administrativos.

- Unidades de cuidados de la comunidad (UCC): compuesto por enfermeros, asistentes sociales, médicos, psicólogos, nutricionistas, fisioterapeutas, logopedas u otros profesionales, en consonancia con las necesidades y la disponibilidad de recursos. Deben prestar cuidados de salud y apoyo psicológico y social de ámbito domiciliario y comunitario a personas de mayor dependencia física y funcional o con enfermedad que requieran acompañamiento próximo y actuación en educación para la salud; su integración en las redes de apoyo a la familia y la implementación de unidades móviles de intervención;

- Unidades de salud pública (USP): no disponen de fisioterapia.

- Unidades de recursos asistenciales compartidos (URAP): prestan servicios de consultoría y asistencia a las unidades funcionales. Compuestas por médicos, asistentes sociales, psicólogos, nutricionistas, fisioterapeutas, logopedas $\mathrm{u}$ otros profesionales pertenecientes a otras unidades funcionales.

Este recién estrenado decreto manifiesta que además pueden existir otras unidades o servicios que sean considerados como necesarios por las administraciones regionales de salud. Y cada unidad funcional presenta un equipo multiprofesional, con autonomía organizativa y técnica, que debe garantizar la cooperación con las demás unidades de funcionamiento de los centros de salud y de los ACES.

Pero, ¿qué han supuesto estos servicios desde que fueron implantados hasta la actualidad y cómo han evolucionado? ¿Cómo han incidido los dispositivos de fisioterapia domiciliaria en cuestiones como la protección social, la salud, la educación, la protección de mayores y personas con discapacidad, la inserción laboral o la adecuación de sus viviendas, más hoy cuando se ha introducido la Ley de la Dependencia y la defensa de estas personas en España ${ }^{16}$ ?

\section{Síntesis de los resultados}

En Portugal nos encontramos con una realidad que nos señala que los fisioterapeutas que trabajan en los centros de salud distribuidos en estas áreas geográficas realizan el tratamiento en poblaciones con elevado ratio, $n .^{\circ}$ de usuarios por fisioterapeuta, y de sus $35 \mathrm{~h} /$ semanales de jornada laboral tienen designadas escasamente $10 \mathrm{~h}$ a la atención domiciliaria, que en muchos de los casos no puede ser llevada a cabo por la gran demanda asistencial que se recibe en los centros ${ }^{15}$. No existe una historia o registro del servicio de fisioterapia consensuada a nivel de todo el territorio portugués, ni protocolos de tratamiento en las patologías que son significativamente más predominantes por la atención realizada. En ocasiones, cada servicio de fisioterapia autónomamente realiza sus registros de información y en otros no existen historias de fisioterapia, con lo que los datos encontrados son difícilmente comparables de unas zonas geográficas a otras.

A lo largo de nuestra búsqueda en las fuentes documentales indicadas, hemos encontrado que en España, el Sistema para la Autonomía y Atención a la Dependencia (SAAD) en julio de 2007 publica un estudio en el que se dice que un total de 52.014 pacientes se han beneficiado en los seis primeros meses del año de los servicios de rehabilitación y fisioterapia ofertados por la Consejería de Salud, tanto en los centros de atención primaria como a través de equipos móviles, según informó la Administración Autonómica Andaluza, dentro de las prestaciones incluidas en el Plan de Apoyo a las Familias. Un total de 4.629 de estas personas fueron asistidas en sus propios domicilios, con una media de diez visitas por paciente ${ }^{17}$.

También existen documentos con información sobre los servicios domiciliarios que prestan fundamentalmente los equipos de atención primaria y atención especializada de soporte en España (Programas de atención domiciliaria equipo de soporte [PADES], Equipos de soporte de atención domiciliaria [ESAD], equipos domiciliarios de los servicios de geriatría hospitalaria, Servicios de ayuda a domicilio [SAD]), pero tras ser analizados, se comprobó que no hacían alusión a la atención fisioterápica ${ }^{5,18}$.

En la actualidad existen muy pocas investigaciones a nivel de la Península Ibérica que ofrezcan datos objetivos basados en la evidencia científica sobre la efectividad de estos servicios de fisioterapia y rehabilitación ofrecidos a domicilio, la satisfacción del usuario y las repercusiones que producen con respecto al tratamiento desarrollado de modo ambulatorio (tabla 2).

Destacamos algunos resultados que nos ofrecen datos, como por ejemplo, que en los pacientes con EPOC la rehabilitación respiratoria ha demostrado, con un alto grado de evidencia, beneficios en la capacidad de esfuerzo y la calidad de vida relacionada con la salud (Regiane, 2007) ${ }^{19}$. Algunos autores como Ariza ${ }^{20}$, en el año 2005, se han referido al servicio de las unidades móviles de fisioterapia describiendo programas de rehabilitación domiciliarios en pacientes con fractura de cadera para determinar si esta intervención multidisciplinaria a domicilio mejora los resultados obtenidos con respecto a una intervención habitual en el hospital. También se ha tratado el tema del paciente geriátrico neurológico en esta unidad de fisioterapia perteneciente a atención primaria (Lozano, 2005) ${ }^{21}$ desde el punto de vista de la adaptación del paciente y sus cuidadores a las condiciones de la vivienda y a la nueva situación a la que se enfrentan, destacando que se evitan los problemas de las barreras arquitectónicas y comorbilidades. Otros autores como los citados en este apartado (Colmenero, Moreno, Muixi, Sánchez, 2006) $)^{22-25}$ han mostrado, a través de comunicaciones en congresos y jornadas de atención 
Tabla 2 Estudios sobre fisioterapia y rehabilitación domiciliaria en la Península Ibérica

\begin{tabular}{|c|c|c|c|c|}
\hline Base de datos & Autor & Año & Tipo de estudio & Resumen de los resultados \\
\hline CUIDEN Plus & $\begin{array}{l}\text { Bocci S, Angelo M } \\
\text { (Brasil) }^{39}\end{array}$ & 2008 & Cualitativo & $\begin{array}{l}\text { La experiencia de cuidadores familiares de } \\
\text { personas con ACV y su relación con el apoyo social } \\
\text { que reciben durante el proceso de rehabilitación } \\
\text { de la persona con ACV en el domicilio }\end{array}$ \\
\hline CUIDEN Plus & Muniz et $\mathrm{al}^{40}$ & 2007 & Descriptivo & $\begin{array}{l}\text { Proyecto de atención interdisciplinaria al anciano } \\
\text { en el nivel primario de la atención: enfoque de } \\
\text { alumnos de fisioterapia }\end{array}$ \\
\hline CUIDEN Plus & Caetano et al (Brasil) ${ }^{41}$ & 2007 & Cualitativo & $\begin{array}{l}\text { La vivencia del proceso de rehabilitación tras ACV. } \\
\text { Identifica los factores que dificultan el proceso de } \\
\text { rehabilitación. }\end{array}$ \\
\hline CUIDEN Plus & $\begin{array}{l}\text { Martínez de la Peña et } \\
\mathrm{al}^{42}\end{array}$ & 2006 & Comunicación & $\begin{array}{l}\text { Estudio de satisfacción de las cuidadoras sobre las } \\
\text { visitas domiciliarias enfermería-fisioterapia en el } \\
\text { Distrito Poniente de Almería }\end{array}$ \\
\hline CUIDEN Plus & Rivas Doblado et $\mathrm{al}^{43}$ & 2004 & Descriptivo & $\begin{array}{l}\text { Muestra una serie de ejercicios rehabilitadores, } \\
\text { durante la estancia hospitalaria del paciente, los } \\
\text { cuales favorecen la recuperación y una favorable } \\
\text { evolución del paciente, en referencia a un grupo } \\
\text { control al que no se sometió a estos ejercicios. }\end{array}$ \\
\hline CUIDEN Plus & Viñas Diz et $\mathrm{al}^{44}$ & 2002 & Descriptivo & $\begin{array}{l}\text { La Escuela universitaria (EU) de Fisioterapia de La } \\
\text { Coruña y la Asociación Coruñesa de Esclerosis } \\
\text { Múltiples (ACEM) ponen en marcha un programa de } \\
\text { voluntariado de tratamiento a domicilio dirigido a } \\
\text { estas personas para mejorar su calidad de vida. }\end{array}$ \\
\hline CUIDEN Plus & Olivan et $\mathrm{al}^{45}$ & 1998 & Revisión & $\begin{array}{l}\text { Una forma de tratamiento en auge: la atención } \\
\text { domiciliaria para discapacitados. Suelen ser } \\
\text { mutuas de seguros o empresas privadas las que } \\
\text { ofrecen estos servicios. }\end{array}$ \\
\hline CUIDEN Plus & $\begin{array}{l}\text { Bernal } E \text {, Faus V, Bernal } \\
\mathrm{E}^{46} \text {. }\end{array}$ & 1997 & Revisión & Enfermería y fisioterapia en atención domiciliaria \\
\hline CUIDEN Plus & Castiella et $\mathrm{al}^{47}$ & 1997 & Revisión & Rehabilitación domiciliaria de la prótesis de cadera \\
\hline CSIC IME & Vilarmau et $\mathrm{al}^{48}$ & 2005 & Descriptivo & $\begin{array}{l}\text { Fractura de fémur del anciano: estudio prospectivo } \\
\text { de las variables asociadas a la prescripción de } \\
\text { rehabilitación hospitalaria versus domiciliaria }\end{array}$ \\
\hline CSIC IME & García M, Abenoza $M^{49}$ & 2004 & Descriptivo & $\begin{array}{l}\text { Analiza a partir de los datos de la red sociosanitaria } \\
\text { catalana, algunos de los aspectos que parecen más } \\
\text { significativos en relación con la provisión del } \\
\text { servicio sociosanitario, el contenido de éste y su } \\
\text { coste financiado. }\end{array}$ \\
\hline ENFISPO & $\begin{array}{l}\text { Pires MJ, Souza Guerra I, } \\
\text { Lopes } A^{50} \text {. }\end{array}$ & 2007 & Descriptivo & $\begin{array}{l}\text { Auditoría a los registros de los fisioterapeutas que } \\
\text { trabajan en la unidad de cuidados intensivos de los } \\
\text { hospitales públicos del área metropolitana de } \\
\text { Lisboa, de acuerdo con los Patrones de Práctica de } \\
\text { la Asociación Portuguesa de Fisioterapeutas }\end{array}$ \\
\hline ENFISPO & $\begin{array}{l}\text { Grupo Investigadores } \\
\mathrm{HC}^{51}\end{array}$ & 2006 & $\begin{array}{l}\text { Estudio analítico } \\
\text { de seguimiento de } \\
\text { cohorte }\end{array}$ & $\begin{array}{l}\text { Seguimiento de una cohorte de atención } \\
\text { domiciliaria. Identifica cuáles son las variables del } \\
\text { paciente y del sercio que recibe que se asocian con } \\
\text { el deterioro funcional y cognitivo. }\end{array}$ \\
\hline ENFISPO & Baño ME, Martínez $\mathrm{P}^{52}$. & 2006 & Revisión & $\begin{array}{l}\text { Valoración domiciliaria del enfermo de Alzheimer: } \\
\text { otro escenario, otro enfoque }\end{array}$ \\
\hline ENFISPO & Grupo ATDOM ${ }^{53}$ & 2003 & Revisión & $\begin{array}{l}\text { Aproximación a la atención domiciliaria: ¿cómo } \\
\text { trabaja la sanidad pública catalana la atención } \\
\text { domiciliaria? }\end{array}$ \\
\hline ENFISPO & Molina MV, Abadía $\mathrm{MJ}^{54}$. & 1993 & Descriptivo & $\begin{array}{l}\text { La asistencia domiciliaria en la rehabilitación de } \\
\text { niños con encefalopatías graves }\end{array}$ \\
\hline Dialnet & Güell Rous $\mathrm{R}^{55}$ & 2007 & $\begin{array}{l}\text { Prospectivo y } \\
\text { aleatorizado }\end{array}$ & $\begin{array}{l}\text { Beneficios de un programa de rehabilitación } \\
\text { respiratoria domiciliaria en pacientes con EPOC } \\
\text { grave }\end{array}$ \\
\hline
\end{tabular}


Tabla 2 (continuación)

\begin{tabular}{|c|c|c|c|c|}
\hline Base de datos & Autor & Año & Tipo de estudio & Resumen de los resultados \\
\hline Dialnet & $\begin{array}{l}\text { Vilarmau M, Nogueras A, } \\
\text { Guirao M, Real J56. }\end{array}$ & 2007 & Descriptivo & $\begin{array}{l}\text { Rehabilitación física de la fractura de fémur en el } \\
\text { anciano: coste y resultados a un año comparando la } \\
\text { rehabilitación hospitalaria con la domiciliaria }\end{array}$ \\
\hline Dialnet & Moya Fernández $L^{57}$. & 2006 & Revisión & Fisioterapia domiciliaria en atención primaria \\
\hline Dialnet & Alonso Valero $D^{58}$. & 2006 & Revisión & $\begin{array}{l}\text { Síndrome de inmovilidad: fisioterapia en el } \\
\text { domicilio }\end{array}$ \\
\hline Dialnet & Arvelo Pérez $\mathrm{I}^{59}$. & 2006 & Comunicación & $\begin{array}{l}\text { Evaluación de la calidad del servicio de ayuda a } \\
\text { domicilio y fisioterapia por parte de los } \\
\text { beneficiarios/as, los cuidadores y el personal de } \\
\text { ayuda }\end{array}$ \\
\hline Dialnet & $\begin{array}{l}\text { Montagut F, Flotats } \mathrm{G} \text {, } \\
\text { Lucas } \mathrm{E}^{3}\end{array}$ & 2005 & Libro & $\begin{array}{l}\text { Rehabilitación domiciliaria. Principios, } \\
\text { indicaciones y programas terapéuticos }\end{array}$ \\
\hline Dialnet & Peña Arrebola $A^{60}$. & 2003 & Revisión & $\begin{array}{l}\text { Papel del ejercicio físico en el paciente con } \\
\text { artrosis. Puede aprovecharse el servicio de } \\
\text { rehabilitación para su mentalización, aprendizaje } \\
\text { y práctica inicial pero se ha demostrado eficacia } \\
\text { aun en los realizados en el domicilio, donde } \\
\text { deberán proseguirse continuadamente. }\end{array}$ \\
\hline Dialnet & Prieto et $\mathrm{al}^{61}$ & 2002 & Cualitativo & $\begin{array}{l}\text { La perspectiva de las cuidadoras informales sobre } \\
\text { la atención domiciliaria. Cómo perciben la calidad } \\
\text { de la atención domiciliaria los cuidadores } \\
\text { familiares de pacientes con cáncer en fase } \\
\text { terminal, ancianos con demencia y personas } \\
\text { intervenidas en programadas de cirugía mayor } \\
\text { ambulatoria }\end{array}$ \\
\hline Dialnet & Díaz de Cerio et al $^{62}$ & 2001 & Descriptivo & $\begin{array}{l}\text { Efecto sobre el consumo de recursos hospitalarios } \\
\text { de un programa de atención geriátrica domiciliaria } \\
\text { en personas ancianas con patología } \\
\text { cardiorrespiratoria muy evolucionada }\end{array}$ \\
\hline Dialnet & Canamasas S, Salas $\mathrm{J}^{63}$. & 1994 & Descriptivo & $\begin{array}{l}\text { Perfil de los pacientes amputados de extremidad } \\
\text { inferior mayores de } 65 \text { años que han realizado } \\
\text { rehabilitación domiciliaria }\end{array}$ \\
\hline TESEO & Pages Bolibar $E^{64}$ & 1995 & Tesis doctoral & $\begin{array}{l}\text { Estudio comparativo entre dos tipos de } \\
\text { rehabilitación para los pacientes ancianos con } \\
\text { fractura de cadera. Resultado funcional y } \\
\text { evaluación económica }\end{array}$ \\
\hline Cochrane Plus & Ashworth et $\mathrm{al}^{65}$ & 2008 & $\begin{array}{l}\text { Revisión Cochrane } \\
\text { traducida }\end{array}$ & $\begin{array}{l}\text { Programas de actividad física en el domicilio versus } \\
\text { en el centro hospitalario para adultos mayores }\end{array}$ \\
\hline Cochrane Plus & $\begin{array}{l}\text { Mottram P, Pitkala K, } \\
\text { Lees } C^{66} \text {. }\end{array}$ & 2008 & $\begin{array}{l}\text { Revisión Cochrane } \\
\text { traducida }\end{array}$ & $\begin{array}{l}\text { Atención crónica institucional versus en el } \\
\text { domicilio para ancianos funcionalmente } \\
\text { dependientes }\end{array}$ \\
\hline Cochrane Plus & $\begin{array}{l}\text { Ward D, Severs M, Dean } \\
\text { T, Brooks } \mathrm{N}^{67} \text {. }\end{array}$ & 2008 & $\begin{array}{l}\text { Revisión Cochrane } \\
\text { traducida }\end{array}$ & $\begin{array}{l}\text { Ámbitos de atención domiciliaria versus } \\
\text { hospitalaria y del propio domicilio para la } \\
\text { rehabilitación de ancianos }\end{array}$ \\
\hline Cochrane Plus & $\begin{array}{l}\text { Handoll HHG, } \\
\text { Sherrington } \mathrm{C}^{68} \text {. }\end{array}$ & 2008 & $\begin{array}{l}\text { Revisión Cochrane } \\
\text { traducida }\end{array}$ & $\begin{array}{l}\text { Estrategias de movilización después de la cirugía } \\
\text { por fractura de cadera en adultos }\end{array}$ \\
\hline Cochrane Plus & $\begin{array}{l}\text { Forster A, Young J, } \\
\text { Langhorne P for the Day } \\
\text { Hospital Group } 69\end{array}$ & 2008 & $\begin{array}{l}\text { Revisión Cochrane } \\
\text { traducida }\end{array}$ & $\begin{array}{l}\text { Atención médica en hospitales de día para ancianos } \\
\text { versus formas alternativas de atención }\end{array}$ \\
\hline Cochrane Plus & $\begin{array}{l}\text { Early Supported } \\
\text { Discharge Trialists }\end{array}$ & 2008 & $\begin{array}{l}\text { Revisión Cochrane } \\
\text { traducida }\end{array}$ & $\begin{array}{l}\text { Servicios destinados a reducir la duración de la } \\
\text { atención en el hospital de los pacientes con ACV } \\
\text { agudo }\end{array}$ \\
\hline $\begin{array}{l}\text { Colegio de } \\
\text { Fisioterapeutas } \\
\text { de Murcia }\end{array}$ & Blanco $A^{71}$ & 2007 & Descriptivo & $\begin{array}{l}\text { "Rehabilitación domiciliaria en pacientes con } \\
\text { fractura de cadera" }\end{array}$ \\
\hline Ovid & $\begin{array}{l}\text { Closa Rusinés } \mathrm{C} \text {, Lucas } \\
\text { Andreu } \mathrm{E} \text {, Montagut } \mathrm{F}^{72}\end{array}$ & 2008 & Descriptivo & $\begin{array}{l}\text { "Indicaciones y criterios de evaluación en } \\
\text { rehabilitación domiciliaria" }\end{array}$ \\
\hline
\end{tabular}


Tabla 2 (continuación)

\begin{tabular}{|c|c|c|c|c|}
\hline Base de datos & Autor & Año & Tipo de estudio & Resumen de los resultados \\
\hline Ovid & $\begin{array}{l}\text { Montoya MD, Ríos-Díaz J, } \\
\text { Martínez-Fuentes } \mathrm{J}^{38} \text {. }\end{array}$ & 2007 & $\begin{array}{l}\text { Descriptivo } \\
\text { retrospectivo }\end{array}$ & $\begin{array}{l}\text { Prevención de las úlceras por presión en pacientes } \\
\text { terminales e inmovilizados seguidos por el equipo } \\
\text { de soporte de atención domiciliaria. Analiza su } \\
\text { aparición y la relación con la atención profesional y } \\
\text { la disponibilidad de medidas de prevención en la } \\
\text { cama y el colchón. }\end{array}$ \\
\hline
\end{tabular}

ACV: accidente cerebrovascular; EPOC: enfermedad pulmonar obstructiva crónica.

primaria relacionadas con el área de Ciencias de la Salud, la variedad de necesidades de la población dependiente que es atendida en este servicio, pero no siempre coinciden en la manera de formularlas ni en las actividades desarrolladas, mostrándonos su diversidad de puntos de vista y procedimientos.

Varios equipos básicos de atención primaria y dispositivos de apoyo a la rehabilitación han hecho acercamientos al estudio de las patologías tratadas en sus servicios, realizando protocolos y pautas de actuación conjuntas en algias vertebrales, tendinitis de hombro, capsulitis adhesiva de hombro, epicondilitis y recuperación del esguince de tobillo. Estos trabajos han servido de guía de procedimientos de las salas de fisioterapia de atención primaria para agilizar la atención coordinada de profesionales mediante pautas comunes, buscando una mayor fiabilidad para futuras investigaciones gracias a la existencia de un sistema de reevaluación eficaz y sencillo de aplicar para valorar los resultados, a reuniones con los profesionales implicados para mejorar la formación, la calidad y la efectividad de la atención, consensuado la aplicación de test funcionales validados al iniciar y al finalizar la atención ${ }^{26,27}$.

En el año 2007, López-Liria ${ }^{7}$ analiza las variables sociodemográficas dependientes del paciente y su patología (edad, sexo, localidad, cuidador principal, proceso discapacitante principal, valoración física y funcional), variables que señalan la independencia del paciente para las actividades de la vida diaria (índice de Barthel) y variables independientes del servicio en la unidad móvil de rehabilitación y fisioterapia en la provincia de Almería (inicio del tratamiento, grupo de atención domiciliaria, motivos de inclusión, objetivos, tratamiento, técnicas) desde el año 2004 hasta el 2007. El objetivo de las actividades desarrolladas en los mayores derivados a este servicio se ha comprobado que no sería tanto el aumento de la expectativa de vida, sino el aumento de la expectativa de vida libre de incapacidad, es decir, prevenir el deterioro funcional $y$, cuando éste se ha producido, recuperar el nivel de función previo con el objetivo de que el anciano pueda permanecer en su domicilio con el mayor grado de independencia posible.

Es también en este año cuando el Departamento de Fisioterapia de la Universidad Católica San Antonio de Murcia publica un artículo basado en un estudio retrospectivo sobre las úlceras por presión y su seguimiento en pacientes atendidos por un equipo de atención domiciliaria del Área II del Servicio Murciano de Salud, y donde se concluye que sería deseable dotar de recursos suficientes tanto a los familiares como a las unidades de atención domiciliaria ${ }^{38}$

Hay algunos estudios a nivel internacional ${ }^{28-30}$ que han evaluado los servicios fisioterapéuticos domiciliarios, ya que aún existen muchos campos y controversias abiertas sin dilucidar en el tema. Se ha demostrado que la prestación de los servicios adecuados con intervenciones específicas para los pacientes geriátricos es efectiva en la reducción de la mortalidad y en la mejora del estado funcional del paciente, así como en la mejora de su ubicación, comportando además una disminución de la utilización de recursos hospitalarios, servicios institucionales o médicos. De hecho, la utilización de servicios sanitarios por parte de las personas mayores guarda más relación con su estado funcional y físico que con su situación económica, comorbilidad o características demográficas. La identificación de los pacientes tributarios de estos servicios así como el desarrollo de estrategias de intervención son, por tanto, claves en la solución de la problemática que afecta a este colectivo ${ }^{31,32}$.

A pesar del beneficio comentado, sólo algunos países de nuestro entorno incluyen expresamente en su sistema de salud la fisioterapia domiciliaria como una prestación con financiación pública. Incluso en los países con mayor tradición, la oferta de estos programas muestra variaciones territoriales importantes y, como consecuencia, sólo una pequeña proporción de los pacientes que se beneficiarían de ella tienen acceso a ésta ${ }^{33,34}$.

\section{Conclusiones}

La involución de la fisioterapia domiciliaria en España y Portugal, o la no implantación sistemática de un mayor número de fisioterapeutas o unidades de rehabilitación hasta el momento actual en los diferentes Departamentos Sanitarios, a pesar del incremento notable de la demanda asistencial y nuevas carteras de servicios, obedece a múltiples causas, si bien la limitación de recursos y la falta de un foro de estudio sobre el tema, entre administración y sociedades científicas de los profesionales implicados podrían explicar esta circunstancia.

Estos estudios son necesarios ya que ofrecen los argumentos a los profesionales sanitarios para la evaluación de estas políticas sanitarias, la distribución adecuada de los recursos para la revisión de los programas, la metodología de actuación, etc. Existe una necesidad de ofrecer un mayor 
protagonismo a las personas, la comunidad y los profesionales, que son los elementos básicos de la reordenación de los servicios ofertados, éstos basados en la calidad, la equidad, la eficiencia, la transversalidad, la participación, la transparencia y la subsidiariedad/sustituibilidad que, entre otros principios básicos, deben configuran el marco general de los modelos sanitarios ${ }^{35}$.

Existiendo estas diferencias relevantes en la oferta real de fisioterapia domiciliaria, tanto entre las comunidades autónomas como dentro de ellas y en ambos países, y teniendo en cuenta que la mayor parte de pacientes posibles candidatos no tiene acceso a ella, la inclusión o expansión de la fisioterapia domiciliaria en atención primaria debe recibir un impulso desde la demanda de la propia sociedad y la evidencia científica de su efectividad y eficacia. Ha de aprovecharse la oportunidad de alcance cuando surge legislación como el reciente Real Decreto que establece la cartera de servicios del Sistema Nacional de Salud o la Ley de Promoción de la Autonomía Personal y Atención a las Personas en Situación de Dependencia que continua desarrollándose. En este campo, los profesionales sanitarios tenemos una posibilidad de poner al servicio de las personas en situación de dependencia y de sus cuidadores nuestros conocimientos y nuestra praxis. Con la Ley de la Dependencia se ha hecho hincapié en reforzar los dispositivos asistenciales para las personas gravemente dependientes, pero no se puede dejar en un segundo plano los esfuerzos por evitar o recuperar dicha dependencia. El objetivo prioritario de la atención a la dependencia no han de ser sólo las personas con dependencia ya establecida, sino también aquellas personas en situación de riesgo y con discapacidad reversible, por lo que las intervenciones que tengan como objetivo la actuación precoz, preventiva y rehabilitadora deben tener un lugar preferente ${ }^{36}$.

La fisioterapia domiciliaria debe dar una respuesta útil, innovadora, eficiente y equitativa ante el gran incremento de demanda de los ciudadanos, como aumento de la esperanza de vida, enfermedades crónicas y disminución de las estancias hospitalarias, utilizando modelos de gestión orientados a la excelencia, procesos asistenciales que garanticen la satisfacción de los enfermos y midan la valoración de los resultados de los objetivos planteados según la patología atendida y el grado de incapacidad funcional de cada paciente.

En la actualidad estamos asistiendo a un proceso donde los profesionales podemos comprobar que la fisioterapia domiciliaria comprende una entidad compleja con unas patologías propias, técnicas y gestión peculiar que requieren consideración y estudio específicos, necesarios y en profundidad. Es fundamental que elaboremos protocolos de prevención y tratamiento para dar cumplida cuenta de lo que se nos exige y que participemos en trabajos de investigación con otros profesionales para obtener datos concretos sobre las enfermedades que despiertan un gran interés clínico sanitario y social, ya que son causa de muerte e incapacitación para los mayores y provoca unos gastos sanitarios muy elevados y difíciles de asumir por el sistema, mutilando en diferente medida la vida plena de un sector de nuestra sociedad. Pero es necesario considerar que también se debe dar una buena respuesta por el impacto que supone en el seno familiar, produciendo en ocasiones un cambio de papeles y desestructuración de la familia ${ }^{37}$.
Por tanto, si se quieren evitar graves desigualdades de acceso a la fisioterapia domiciliaria y promover una atención de calidad, es necesaria una actuación conjunta de las administraciones y las sociedades científicas, y una mayor concienciación de los profesionales para promover y garantizar su desarrollo ordenado y beneficioso. Un moderno sistema de atención de la salud debe reconocer a las personas mayores como sus principales usuarios, a la vez que garantizar la búsqueda activa de la enfermedad, reconocer sus distintas y específicas manifestaciones, anticipar y posponer la posible dependencia y disponer de la tecnología e información científica adecuada para estos fines a través de los profesionales sanitarios que posee y que están cualificados para ello. Se debe buscar la potenciación de los programas de atención domiciliaria como una opción asistencial que proporcione a los ancianos dependientes una mejor calidad de vida: menores complicaciones graves, disminución de la institucionalización, menor número de reingresos, mejora de la información y la relación médicopaciente, profesionales sanitarios-familia, etc., garantizando además la continuidad asistencial.

Los equipos de fisioterapia domiciliaria deben continuar presentado una respuesta rápida a las situaciones que suelen plantarse, sin descuidar el control del paciente tras el alta hospitalaria y la alteración sintomática severa o la recaída tras un proceso de recuperación.

Concluimos diciendo que se debe apostar por esta iniciativa y hacerla extensible en lo posible a la mayoría de las patologías que provocan dependencia, sin quedarse estancada como una iniciativa puntual que tuvo lugar con una política sanitaria del momento.

\section{Conflicto de intereses}

Esta investigación ha recibido la financiación para la realización de una estancia de excelencia en la Universidad de Lisboa (Portugal) dentro de las ayudas de la Junta de Andalucía y la Universidad de Almería. No existe una asociación comercial que pueda dar lugar a un conflicto de intereses en relación con el artículo remitido.

\section{Agradecimientos}

Este artículo ha sido elaborado como parte del trabajo de una beca de investigación del Plan Propio de la Universidad de Almería (Estancias de Excelencia en Centros Extranjeros), así como gracias a la subvención concedida por la Consejería de Innovación, Ciencia y Empresa de la Junta de Andalucía, de acuerdo con lo establecido en el Decreto 245/ 1997, del 15 de octubre, (BOJA núm. 130, de 8 de noviembre).

\section{Bibliografía}

1. Resolución de la Gerencia de Atención Especializada, Área 4 de Madrid, por la que se anuncia la adjudicación de PNSP derivado de contrato marco de ref. CMR Carrasco 2000: 02/2 concierto para rehabilitación domiciliaria del Hospital "Ramón y Cajal". Real Decreto 2/2000, de 16 de junio, art. 93.2. Madrid. Consultado el (13-06-2006) en http://www.cde.ua.es/boe/ 20020102.htm 
2. Organización Mundial de la Salud (OMS). Séptima Conferencia Mundial sobre el Envejecimiento organizada por la Federación Internacional sobre el Envejecimiento. 2004 Sep 6; Ginebra [consultado el: 05 de marzo de 2006] Disponible en: http:// www.who.int/mediacentre/news/releases/2004/pr60/es/

3. Montagut F, Flotats G, Lucas E. Rehabilitación Domiciliaria. Principios, indicaciones y programas terapéuticos. Barcelona: Masson; 2005.

4. IMSERSO. Las personas mayores en España. INFORME 2006. [citado 10 Jun 2007]. Disponible en: URL: http://www.segsocial.es/imserso/

5. Informe de la Sociedad Española de Geriatría y Gerontología. Análisis y evaluación de la red de servicios sanitarios dedicados a la dependencia: programas de prevención, atención domiciliaria y hospitalización. 2005. [citado 3 Jun 2007]. Disponible en: URL: http://www.imsersomayores.csic.es/documentacion/ documentos/registro.jsp?id=1153

6. López-Liria R, Fernández M, Sáez C, Rodríguez C. Atención integral del Alzheimer en el Hospital de Día. En: Gázquez JJ, Castellón A, Pérez MC, Yuste N, editors. Valoración y Tratamiento de las demencias. Apoyo a cuidadores. 1st ed. Almería: Universidad de Almería; 2006. p. 153-67.

7. López-Liria R. Estudio descriptivo de las patologías tratadas por las Unidades Móviles de Fisioterapia en la provincia de Almería: su influencia en la independencia funcional de la población geriátrica. Memoria docente e investigadora del Programa de Doctorado Gerontología y Geriatría. Universidad de Almería. Diciembre 2007.

8. Plan de Rehabilitación de la Comunidad Valenciana. Generalitat Valenciana. Valencia: Consellería de Sanitat i Consum; 1988.

9. Generalitat Valenciana. Conselleria de Sanitat. Plan de Rehabilitación de la Comunidad Valenciana. Programa Especial de Rehabilitación. Valencia: Conselleria de Sanitat, Generalitat Valenciana; 1991. Serie: Programas Especiales N² 2. 1991.

10. Plan de Rehabilitación de Castilla-La Mancha'04. Castilla-La Mancha: Servicio de Salud de Castilla- La Mancha. 2004.

11. Documento de trabajo del Servicio Andaluz de Salud (Agosto 2002). Estrategias de mejora para la atención rehabilitadora y fisioterapéutica en Andalucía. Equipos móviles. Plan de Atención a la Familia. Dirección General de Asistencia Sanitaria. Sevilla: Consejería de Salud, Junta de Andalucía: 2002.

12. Decreto $137 / 2002$ de 30 de abril. Fisioterapia y Rehabilitación en Atención Primaria de Salud. Ampliación. BOJA N. ${ }^{\circ} 56$. Sevilla: Consejería de Salud, Junta de Andalucía: 2004. p. 7043.

13. Rehabilitación y fisioterapia en Atención Primaria. Guía de procedimientos. Sevilla: Servicio Andaluz de Salud; 2003.

14. Ministerio da Saúde. Decreto- Ley N.o 28/2008, del 22 de Febrero.

15. Saude e Apoyo Social. Relatório de Monitorizaçao da implementaçao das experiencias piloto da rede nacional de cuidados continuados integrados (RNCCl). Lisboa; Ministerio da Saude e Apoyo Social, Lisboa, Portugal: 2007.

16. Ley $39 / 2006$, de 14 de diciembre, de Promoción de la Autonomía Personal y Atención a las personas en situación de dependencia; Ministerio da Saude e Apoyo Social, Lisboa, Portugal: 2007. [citado 16 Jun 2007]. Disponible en: URL: http://www.boe.es/boe/dias/2006/12/15/pdfs/A44142-44156. pdf

17. Sistema de Información del Sistema para la Autonomía y Atención a la Dependencia. Ministerio de Trabajo y Asuntos Sociales. - Secretaría de Estado de Servicios Sociales, Familias y Discapacidad - IMSERSO. 2007. [citado 7 Feb 2007]. Disponible en: URL: http://www.seg-social.es/imserso/dependencia/dep_ laad.html

18. Rodríguez R. Los servicios de ayuda a domicilio: Planificación y gestión de casos: manual de formación para auxiliares. Madrid: Panamericana; 2003.
19. Regiane V, Gorostiza A, Gáldiz J, López de Santa María E, Casan $P$, Güell R. Beneficios de un programa de rehabilitación respiratoria domiciliaria en pacientes con EPOC grave. Arch Bronconeumol. 2007;43:599-604.

20. Ariza MP, Anula AM, Anula J, Álamo A. Programa de rehabilitación domiciliario en paciente de fractura de cadera. Comunicación presentada en II Jornadas Científicas de Ciencia de la Salud. Escuela Universitaria de Ciencias de la Salud Granada: Universidad de Granada; 2005.

21. Lozano JM, Ruiz G, Rodríguez M, Álamo A. Tratamiento Fisioterápico del paciente geriátrico neurológico en Atención Primaria. Comunicación presentada en II Jornadas Científicas de Ciencia de la Salud. Escuela Universitaria de Ciencias de la Salud Granada: Universidad de Granada; 2005.

22. Colmenero I, Gallo S, Sánchez M, Moreno D. La unidad móvil de Fisioterapia de Jaén: cuatro años apoyando a las familias andaluzas. Comunicación presentada a la IX Jornadas Nacionales de Fisioterapia en Atención Primaria. Murcia: Universidad de Murcia; 2006. Disponible en: http://www.um.es/estudios/ cursos/jornadas/IXAP/AEF/horarios-carteles.pdf Consultado el 10-6-2006.

23. Moreno D, Sánchez M, Colmenero I, Gallo S. Mejora en la independencia de la movilidad y el cuidado personal, obtenida por los pacientes atendidos por la unidad móvil de Fisioterapia de Jaén. Comunicación presentada a la IX Jornadas Nacionales de Fisioterapia en Atención Primaria. Murcia: Universidad de Murcia; 2006. Disponible en: http://www.um.es/estudios/ cursos/jornadas/IXAP/AEF/horarios-carteles.pdf Consultado el 10-6-2006.

24. Muixi R, Maya A, Jové J, Inglés Mạ.M. Comparativa entre Atención Domiciliaria Urbana y Atención Domiciliaria Rural. Comunicación presentada a la IX Jornadas Nacionales de Fisioterapia en Atención Primaria. Murcia: Universidad de Murcia; 2006. Disponible en: http://www.um.es/estudios/ cursos/jornadas/IXAP/AEF/horarios-carteles.pdf Consultado el 10-6-2006.

25. Sánchez M, Moreno D, Gallo S, Colmenero IM. El paciente en la Unidad Móvil de Jaén: características que lo definen. Comunicación presentada a la IX Jornadas Nacionales de Fisioterapia en Atención Primaria. Murcia: Universidad de Murcia; 2006. Disponible en: http://www.um.es/estudios/cursos/jornadas/ IXAP/AEF/horarios-carteles.pdf Consultado el 10-6-2006.

26. Consejería de Salud. Control y mejora continua de los Procesos Asistenciales Integrados. En: Consejería de Salud. Guía de diseño y mejora continua de Procesos Asistenciales; Sevilla: Consejería de Salud, Junta de Andalucía; 2001. p. 83-96.

27. Servicio Andaluz de Salud. Cuestionarios, tests e índices para la valoración del paciente. 2 ed. Sevilla: Editado por la Consejería de Salud, Junta de Andalucía; 2004. [citado 9 Jun 2007]. Disponible en: URL: http://www.infodisclm.com/documentos/ salud/cuestionario.pdf

28. Mayo N, Wood-Dauphinee S, Cotê R, Gayton D, Carlton J, Buttery J, et al. There's no place like home: an evaluation of early supported discharge for stroke. Stroke. 2000;31:1016-23.

29. Chimenti CE, Ingersoll G. Comparison of home health care physical therapy outcomes following total knee replacement with and without subacute rehabilitation. J. Geriatr Phys Ther. 2007;30(3):102-8.

30. Mahomed NN, Koo Seen Lin MJ, Levesque J, Lan S, Bogoch ER. Determinats ad outcomes of inpatient versus home based rehabilitation following elective hip and knee replacement. J Rehumatol. 2000;27:1753-8.

31. Crespo M, López J. El apoyo a los cuidadores de familiares mayores dependientes en el hogar: desarrollo del programa "Cómo mantener su bienestar". Premio IMSERSO "Infanta Cristina". Madrid: Ministerio de Trabajo y Asuntos Sociales; 2006. 
32. Sociedad Española de Medicina Geriátrica (SEMEG). Prevención de la dependencia en las personas mayores. Madrid: SEMEG; 2006.

33. Leichsenring K. Providing integrated health and social care for older persons - A European overview. European Centre for Social Welfare Policy and Research; 2003.

34. Lopes MI. Orientações da Política de Cuidados às Pessoas Idosas e Dependentes Modelo de Cuidados em Portugal e nalguns Países Europeus. I Congresso Internacional de Gerontologia: Viver para sempre. Odivelas; 2006.

35. Have $S$ ten, Have $\mathrm{W}$ ten, Stevens F. Lo más importante de la gestión empresarial: las herramientas y prácticas de gestión que mejorarán su empresa. Barcelona: Deusto; 2004.

36. Opinión. Fisioterapia, profesión de referencia ante la ley de dependencia. El fisioterapeuta. Boletín informativo periódico $\mathrm{n}^{\circ}$ 132. Ed. Asociación Española de Fisioterapeutas: 2007.

37. Gómez MA, Martos A, Castellón A. La capacidad funcional y el apoyo social informal en un grupo de personas mayores institucionalizadas. Rev Mult Gerontolog. 2005;15:85-91.

38. Montoya MD, Ríos-Díaz J, Martínez-Fuentes J. Prevención de las úlceras por presión en pacientes terminales e inmovilizados seguidos por el equipo de soporte de atención domiciliaria. Revista Esp Geriatria y Gerontología. 2007;42:263-70.

39. Bocchi S, Angelo M. Entre la libertad y la reclusión: el apoyo social como un componente de la calidad de vida del binomio cuidador familiar y persona dependiente. Rev Latino-am Enfermagem -Bra. 2008;16:15-23.

40. Muniz C, Arnaut A, Yoshida M, Trelha C, Dellaroza M. Proyecto de atención interdisciplinar al anciano en el nivel primario de la atención: enfoque de alumnos de fisioterapia]. Revista APS Bra. 2007;10:84-9.

41. Caetano J, Damasceno M, Soares E, Fialho A. A vivência do processo de reabilitação após acidente vascular cerebral: um estudo cualitativo. OBJN. 2007;6.

42. Martínez R, Navarro L, Téllez R, Pérez MB. Estudio de satisfacción de las cuidadoras, sobre las visitas domiciliarias enfermería-fisioterapia en el Distrito Poniente de Almería. Evidentia. 2006;Año 3.

43. Rivas JS, Soriano F, Mejías F. Ejercicios de rehabilitación en la Meniscectomía artroscópica. Hygia de Enfermería. 2004;Año $\mathrm{XVI}: 5-9$.

44. Viñas S, Martínez A, Martínez S, Raposo I, Fernández R. Abordaje de la esclerosis múltiple desde la atención primaria. Cuest Fisioterapia. 2002;21:83-9.

45. Oliván B, Franco MA, Domínguez MP. Una forma de tratamiento en auge: la atención domiciliaria para discapacitados. Anal Cienc Salud. 1998;1:123-32.

46. Bernal E, Faus V, Bernal E. Enfermería y fisioterapia en atención domiciliaria. Enferm Integral. 1997;40:XVII-XX.

47. Castiella S, Lión S, Someso E, Figueroa J, Bañales T. Rehabilitación domiciliaria de la prótesis de cadera. Cad Aten Primaria. 1997;4:195-201.

48. Vilarmau M, Nogueras A, Guirao M, Real J, López M, Bacos C. Fractura de fémur del anciano: estudio prospectivo de las variables asociadas a la prescripción de rehabilitación hospitalaria versus domiciliaria. Revista Multidisciplinar de Gerontología. 2005; 15: 81-84.

49. García M, Abenoza M. Consideraciones para el análisis costebeneficio en la red sociosanitaria. Rehabilitación. 2004;38: 362-7.

50. Pires MJ, Souza I, Lopes A. Auditoría a los registros de los fisioterapeutas que trabajan en la Unidad de Cuidados Intensivos de los hospitales públicos del área metropolitana de Lisboa, de acuerdo con los Patrones de Práctica de la Asociación Portuguesa de Fisioterapeutas. Revista Iberoamericana de Fisioterapia y Kinesiología. 2007;10:86-96.

51. Grupo Investigadores HC. Seguimiento de una cohorte de atención domiciliaria. Atención primaria. 2006;38:47-50.
52. Baño ME, Martínez P. Valoración domiciliaria del enfermo de Alzheimer: otro escenario, otro enfoque. Revista de Fisioterapia. 2006;5:3-9.

53. Societat Catalana de Medicina Familiar y Comunitaria. Grupo ATDOM. Aproximación a la atención domiciliaria: ¿cómo trabaja la sanidad pública catalana la atención domiciliaria? Atención primaria. 2003;31:473-9.

54. Molina MV, Abadía MJ. La asistencia domiciliaria en la rehabilitación de niños con encefalopatías graves. Revista: Medicina de Rehabilitación. 1993; VI(2):20-4.

55. Güell Rous R. Beneficios de un programa de rehabilitación respiratoria domiciliaria en pacientes con EPOC grave. Archivos de bronconeumología. 2007;43:599-604.

56. Vilarmau M, Nogueras A, Guirao M, Real J. Rehabilitación física de la fractura de fémur en el anciano: coste y resultados a un año comparando la rehabilitación hospitalaria con la domiciliaria. Revista multidisciplinar de gerontología. 2007;17:187-91.

57. Moya L. Fisioterapia domiciliaria en atención primaria. Jano: Medicina y humanidades. 2006;1600:41.

58. Alonso D. Síndrome de inmovilidad: fisioterapia en el domicilio. Jano: Medicina y humanidades. 2006;1611k:43.

59. Arvelo IC. Evaluación de la calidad del Servicio de ayuda a domicilio y fisioterapia por parte de los beneficiarios/as, los cuidadores y personal de ayuda. Trabajo social y salud. 2006;54:183-92.

60. Peña A. Papel del ejercicio físico en el paciente con artrosis. Rehabilitación: Revista de la Sociedad Española de Rehabilitación y Medicina Física. 2003;37:307-22.

61. Prieto MA, Gil E, Hederle C, Frías A. La perspectiva de las Cuidadoras informales sobre la atención domiciliaria. Un estudio cualitativo con ayuda de un programa Informático. Rev. Esp. Salud Pública. 2002;75.

62. Díaz MA, Gómez C, Gómez M, Mareque M, Pérez A, Rol de la Morena MJ. Efecto sobre el consumo de recursos hospitalarios de un programa de atención geriátrica domiciliaria en personas ancianas con patología cardiorrespiratoria muy evolucionada. Revista española de salud pública. 2001;75:559-67.

63. Canamasas S, Salas J. Perfil de los pacientes amputados de extremidad inferior mayores de 65 años que han realizado rehabilitación domiciliaria. Revista de gerontología. 1994;4: 25-9.

64. Pages Bolibar E. Estudio comparativo entre dos tipos de rehabilitación para los pacientes ancianos con fractura de cadera. Resultado funcional y evaluación económica [tesis doctoral]. Barcelona: Universidad Barcelona; 1995.

65. Ashworth NL, Chad KE, Harrison EL, Reeder BA, Marshall SC. Programas de actividad física en el domicilio versus en el centro hospitalario para adultos mayores (Revisión Cochrane traducida). En: La Biblioteca Cochrane Plus, número 3, 2008. Oxford, Update Software Ltd. [consultado el 13 de agosto de 2008] Disponible en: URL: http://www.update-software.com

66. Mottram P, Pitkala K, Lees C. Atención institucional versus domiciliaria a largo plazo para ancianos con dependencia funcional (Revisión Cochrane traducida). En: La Biblioteca Cochrane Plus, número 3, 2008. Oxford, Update Software Ltd. Disponible en: URL: http://www.update-software.com

67. Ward $D$, Severs $M$, Dean $T$, Brooks $N$. Ámbitos de atención domiciliaria versus hospitalaria y del propio domicilio para la rehabilitación de ancianos (Revisión Cochrane traducida). En: La Biblioteca Cochrane Plus, número 3, 2008. Oxford, Update Software Ltd. [consultado el 13 de agosto de 2008] Disponible en: URL: http://www.update-software.com

68. Handoll HHG, Sherrington C. Estrategias de movilización después de la cirugía por fractura de cadera en adultos (Revisión Cochrane traducida). En: La Biblioteca Cochrane Plus, número 3, 2008. Oxford, Update Software Ltd. [consultado el 13 de agosto de 2008] Disponible en: URL: http://www.updatesoftware.com 
69. Forster A, Young J, Langhorne P for the Day Hospital Group. Atención médica en hospitales de día para ancianos versus formas alternativas de atención (Revisión Cochrane traducida). En: La Biblioteca Cochrane Plus, número 3, 2008. Oxford, Update Software Ltd. [consultado el 13 de agosto de 2008] Disponible en: URL: http://www.update-software.com

70. Early Supported Discharge Trialists. Servicios destinados a reducir la duración de la atención en el hospital de los pacientes con accidente cerebrovascular agudo (Revisión
Cochrane traducida). En: La Biblioteca Cochrane Plus, número 3, 2008. Oxford, Update Software Ltd. Disponible en: [consultado el 13 de agosto de 2008] URL: http://www.updatesoftware.com

71. Blanco A. Rehabilitación domiciliaria en pacientes con fractura de cadera. Fisioterapia y Calidad de Vida. 2007;10.

72. Closa C, Lucas E, Montagut F. Indicaciones y criterios de evaluación en rehabilitación domiciliaria. Formación Médica Continuada en Atención Primaria. 2008;15. 